\title{
Fast and accurate earthquake location within complex medium using a hybrid global- local inversion approach*
}

\author{
Chaoying Bai ${ }^{1,2, *}$ Rui Zhao ${ }^{1}$ and Stewart Greenhalgh ${ }^{3,4}$ \\ ${ }^{1}$ Department of Geophysics, College of Geology Engineering and Geomatics, Chang'an University, \\ Xi' an 710054, China \\ ${ }^{2}$ Key Laboratory of Western China's Mineral Resources and Geology Engineering, Ministry of Education, \\ Xi' an 710054, China \\ ${ }^{3}$ Institute of Geophysics, ETH Zürich CH 8092, Switzerland \\ ${ }^{4}$ Department of Physics, University of Adelaide, Adelaide SA 5005, Australia
}

\begin{abstract}
A novel hybrid approach for earthquake location is proposed which uses a combined coarse global search and fine local inversion with a minimum search routine, plus an examination of the root mean squares (RMS) error distribution. The method exploits the advantages of network ray tracing and robust formulation of the Fréchet derivatives to simultaneously update all possible initial source parameters around most local minima (including the global minimum) in the solution space, and finally to determine the likely global solution. Several synthetic examples involving a 3-D complex velocity model and a challenging source-receiver layout are used to demonstrate the capability of the newly-developed method. This new global-local hybrid solution technique not only incorporates the significant benefits of our recently published hypocenter determination procedure for multiple earthquake parameters, but also offers the attractive features of global optimal searching in the RMS travel time error distribution. Unlike the traditional global search method, for example, the Monte Carlo approach, where millions of tests have to be done to find the final global solution, the new method only conducts a matrix inversion type local search but does it multiple times simultaneously throughout the model volume to seek a global solution. The search is aided by inspection of the RMS error distribution. Benchmark tests against two popular approaches, the direct grid search method and the oct-tree important sampling method, indicate that the hybrid global-local inversion yields comparable location accuracy and is not sensitive to modest level of noise data, but more importantly it offers two-order of magnitude speed-up in computational effort. Such an improvement, combined with high accuracy, make it a promising hypocenter determination scheme in earthquake early warning, tsunami early warning, rapid hazard assessment and emergency response after strong earthquake occurrence.
\end{abstract}

Key words: complex velocity model; SPM ray tracing; matrix inversion; earthquake location; a global solution; RMS error inspection

CLC number: P315.3 Document code: A

\section{Introduction}

Earthquake location is a non-linear inverse problem (Thurber, 1985; Thurber and Rabinowitz, 2000) and still a fundamental issue in seismology, especially for complex 3-D models. The current location methodologies can be grouped into two main categories: the linearized

\footnotetext{
* Received 31 August 2009; accepted in revised form 14 September 2009; published 10 October 2009.

-Corresponding author.e-mail: baicy@chd.edu.cn
}

inversion approaches and the direct global search methods. For weakly non-linear problems, the most popular linearized earthquake location programs used today on a regional or global scale include HYPO71 (Lee and Lahr, 1975), HYPOINVERSE (Klein, 1978, 1989), HYPOCENTER (Lienert et al, 1986), plus revisions thereafter (i.e., Pavlis and Booker, 1980), and the double-difference earthquake location algorithm (DDEL, Waldhauser and Ellsworth, 2000). They considered only a 1-D stratified velocity model. Whilst there exist several methods for hypocenter determination on a local scale in 3-D veloc- 
ity models, for example, the multiple earthquake location approach (MEL) of Bai and Greenhalgh (2006), the conversion from a 1-D to a 3-D velocity model makes the location process more complex and the problem far more non-linear in nature. Furthermore, all linearized inversion procedures used in earthquake location depend not only on the correct velocity model to be used, but also on satisfactory initial values for the source parameters. This leads to a local minimization solution in most 3-D cases. To date it has been impossible to find the true global solution in the linearized matrix inversion procedure. This presents a real challenge in seismology and geophysics in general.

For highly non-linear 3-D event location problems the usual approach is to use a Monte Carlo-type global search method. There are several variants on this approach: (1) regular and deterministic search, such as grid-search, nested grid-search and 'crude' Monte-Carlo search (e.g., Nelson and Vidale, 1990; Sambridge and Mosegaard, 2002), which use a global and well-distributed sampling of the model space and thus may estimate the complete location; (2) directed search, that is stochastic search techniques including evolutionary, adaptive global search methods such as genetic algorithms (e.g., Kennett and Sambridge, 1992) and simulated annealing (e.g., Tarantola, 2005); (3) importance sampling, in which the estimated properties of a target (misfit or likelihood) function can be increased by choosing a sampling density which follows the target function as closely as possible (e.g., Lepage, 1978). The importance sampling techniques may be used to find complete, probabilistic solutions to inverse problems. They include the metropolis algorithm (e.g., Mosegaard and Tarantola, 1995), the neighborhood algorithm (e.g., Sambridge, 1999a, b; Sambridge and Kennett, 2001) and the oct-tree algorithm (e.g., Lomax and Curtis, 2001).

The optimal Monte Carlo-type global search approaches are advantageous over the linearized inversion procedure in the sense of solution accuracy (i.e., global solution), but they are still time consuming in some problems. This can be an issue in certain applications like earthquake early warning, tsunami early warning, rapid hazard assessment and emergency response after strong earthquake occurrence, where CPU time is a critical factor for method selection. Because of the disadvantages of the single approach, there are also some kinds of combination of the two approaches (the global search and the local inversion), for example, a hybrid global search algorithm (or hybrid global optimization algorithm) is to use a combination of simulated annealing (global search) and downhill simplex method (local inversion) (e.g., Hartzell and Liu, 1995, 1996; Fallat and Dosso, 1999). Here, downhill simplex (local) and simulated annealing (global) methods are developed individually and combined to produce a hybrid simplex simulated annealing algorithm. Therefore the question, which we wish to investigate in this paper, is whether some novel adaptation to the linearized matrix inversion approach (local search minimization) to earthquake location can be devised to seek the global solution in an efficient and effective manner over the global grid search approach. In other words, is it possible to select the initial values by sampling the whole solution space so as to cover all or most local (including global) minima, and still avoid the time-consuming Monte Carlo-like approach? The answer is yes, as shown below. The new approach builds on some novel features incorporated into a recently reported hypocenter procedure developed by Bai and Greenhalgh (2006) in which there is no need for iterative ray tracing, the formulation of the Jacobian matrix for updating the hypocentral co-ordinates is simple and robust, and the non-linear solver can be used to locate numerous events in one run of the program.

In the next section of this paper we first review the ideas behind the new development. And then we describe the procedure in detail. Finally we present several numerical examples of using the new location procedure in a complex 3-D model and show its good performance, even for challenging situations where the events lie far outside the seismic network. Furthermore, benchmark tests are provided against the direct grid search method (Nelson and Vidale, 1990) and the oct-tree algorithm (Lomax and Curtis, 2001) in the sense of location accuracy, computational efficiency and sensitivity to noise data. The new method is shown to compare favorably in both location accuracy and computational efficiency, more importantly it is less sensitive to noise data.

\section{New procedure}

Figure 1 illustrates the fundamental issue of non-linear behavior with an inversion routine. There exists a global minimum to the misfit function (or RMS error) between the calculated and the observed data, and numerous local minima in this model space (see Figure 1). In a matrix inversion approach to earthquake location, the initial parameters (source position and origin time) are critical if it leads to a global solution. If the initial 
source parameters are far removed from the true values (i.e., at the wrong place), we only find a local but not a global solution using a local minimization procedure (e.g., steepest descent, conjugate gradient and etc). For example, in Figure 1 if the initial set of model parameters lies on the limb of a local minimum, using a local inversion routine will only find the bottom of that particular valley. Certainly there are some kinds of perturbation methods that allow a jump from one local minimum to another one nearby. It may take a long time to determine all local minima (or all local maxima instead, if the cost-function is defined differently). Sometimes it is impossible to jump to the nearby global minimum when the solution space is more complex or exhibits highly non-linear behavior or if à priori information is lacking. To overcome such deficiencies it is possible to sample the solution space with a modest number of starting values (initial source positions) so that they may bracket any local (including global) minima. This will only be efficient if one does not need to repeat the travel time computations for every such starting position. In such a situation a matrix inversion style local minimization procedure is possible to find the global solution by virtue of having at least one of the initial sets of starting source parameters near the global solution in the model space.



Figure 1 A scheme of error variation with solution parameters indicating the complexity of non-linear behavior. There are multiple local minima, and the initial selection of parameters may only converge to the adjacent local minimum and not the global minimum.

With traditional hypocenter determination algorithms ray tracing is performed using the shooting or bending method. It must be repeated for every trial source position for every update from a given starting position. The CPU time increases according to the inverse scale length used to sample the solution space. A very uniformly dense grid (or random walk) of starting positions again leads to the Monte Carlo style global inversion so that a prohibitive amount of CPU time is involved.

An alternative to such an approach is to use a grid-cell based algorithm, such as the finite difference eikonal equation solver (Vidale, 1988, 1990) or the shortest path method (SPM, Moser, 1991; MSPMmodified SPM, Bai et al, 2007) to determine the travel times. The grid-cell based ray tracing methods offer a major advantage in accomplishing the above task. They calculated first arrival times (later arrivals are also possible, see Rawlinson and Sambridge, 2004; Bai et al, 2009) and the related ray paths on all grid nodes gradually, as the wave field evolves from the source outward. This makes multiple earthquake locations (MEL) possible in one simultaneous execution of the algorithm but with far less CPU time consumption. This was shown in the recent paper by Bai and Greenhalgh (2006), in which they presented an accurate and efficient procedure for determining local earthquake parameters (including source co-ordinates and origin time) in complex 3-D models. They used a MSPM method which can employ relatively coarse primary node spacing on the cells, but with secondary nodes distributed over the cell surfaces to improve ray angular coverage and hence the accuracy of the travel times. Velocity values at the secondary nodes and at source and receiver locations (if they do not coincide with primary nodes, e.g., they can be inside the cells) are calculated by interpolation using a Lagrangian tri-linear function linked to the sampled velocity values at the primary nodes. Details on the MSPM ray tracing are given by Bai et al (2007).

The local minimization part of the non-linear inversion to obtain the hypocenter can be formulated as a damped minimum norm, constrained least squares problem (DMNCLS) where one seeks to minimize the function (Zhou et al, 1992):

$$
\frac{1}{2}\left[\|\delta \boldsymbol{r}-A \delta \boldsymbol{x}\|_{L_{2}}^{2}+\mu\|\delta \boldsymbol{x}\|_{L_{2}}^{2}\right], \quad \boldsymbol{a} \leq \delta \boldsymbol{x} \leq \boldsymbol{b} .
$$

The squared norm in each case is defined by $\|\boldsymbol{s}\|_{L_{2}}^{2}=\boldsymbol{s}^{\mathrm{T}} \boldsymbol{W} \boldsymbol{s}$ where the quantity $\boldsymbol{s}$ is used to represent either of the two terms given previously. The quantity $\boldsymbol{W}$ is a positive symmetric weighting matrix operator that is set separately for either the data space (first term $\delta$ ) or the model space (second term, $\delta \boldsymbol{x}$ in this case). The quantity $\mu$ is a (soft) damping factor, and $\boldsymbol{a}$ and $\boldsymbol{b}$ are (hard) constraint bounds (upper and lower limits, respectively) on the solution space of the updated source 
parameters after inversion, so that the source parameters must lie within a stipulated range (say, position is within the model and origin time is equal or larger than $0.0 \mathrm{~s}$ ).

The residual at the $i$ th station is actually formed as the difference between the observed travel times (that is arrival time $t_{\mathrm{obs}}$ minus origin time $t_{0}$ ) and the theoretically calculated time $t_{\text {cal }}$ according to the expression $\delta r^{i}=t_{\text {cal }}^{i}+t_{0}^{i}-t_{\mathrm{obs}}^{i}$. The arrival time residual $\delta r^{i}$ can also be expressed in terms of the partial derivatives with respect to the hypocentral parameters $\left(x, y, z, t_{0}\right)$ :

$$
\begin{gathered}
\delta r^{i}=\frac{\partial t_{\mathrm{cal}}^{i}}{\partial x} \delta x+\frac{\partial t_{\mathrm{cal}}^{i}}{\partial y} \delta y+\frac{\partial t_{\mathrm{cal}}^{i}}{\partial z} \delta z+ \\
\frac{\partial t_{\mathrm{cal}}^{i}}{\partial t_{0}} \delta t, \quad i=1,2, \cdots, n .
\end{gathered}
$$

Since observed times are fixed, the derivatives of the residuals $r$ are simply the derivatives of $t_{\text {cal }}$ evaluated at the current assumed source position and origin time.

In matrix notation equation (2) takes the following form:

$$
\delta \boldsymbol{r}=\boldsymbol{A} \delta \boldsymbol{x}
$$

where $\delta \boldsymbol{r}=\left(\delta r^{1}, \delta r^{2}, \cdots, \delta r^{n}\right), \delta \boldsymbol{x}=(\delta x, \delta y, \delta z, \delta t)^{\mathrm{T}}$ and $\boldsymbol{A}$ is the analytic Jacobian matrix of partial derivatives of time with respect to hypocentral co-ordinates and origin time.

Adjustments $(\delta x, \delta y, \delta z, \delta)$ are then made to the source parameters through minimizing equation (1). These values are added to (or subtracted from) the initial or previous hypocentral co-ordinates and origin time to obtain the updated source parameters $\left(x_{k}, y_{k}, z_{k}, t_{k}\right)$ viz, $\delta x=x_{k}-x_{0}, \delta y=y_{k}-y_{0}, \delta z=z_{k}-z_{0}$ and $\delta t=t_{k}-t_{0}$.

With an iterative approach one can easily obtain the improved (final approximated) earthquake parameters $\left(x_{f}, y_{f}, z_{f}, t_{f}\right)$ by continually updating the source parameters $\left(x_{k}, y_{k}, z_{k}, t_{k}\right)$. The Jacobian needs to be redefined at each step of the process because the derivatives are evaluated for the current values of the source. Iterative inversion requires knowledge of the travel times and elements of the Jacobian matrix viz, the travel time derivatives with respect to the source parameters changes at a trial (updated) hypocenter.

Bai and Greenhalgh (2006) calculated the Jacobian $\boldsymbol{A}$ in a particularly simple and robust way. They placed six additional candidate source positions around the current trial hypocenter, in the positive and negative $x, y$ and $z$ directions. Invoking reciprocity, the MSPM gives the travel times from each receiver (treated as a source) to all $3 \mathrm{D}$ node points within the model. It also allows one to calculate travel times (by interpolation) to any interior position in a given cell. Therefore it is a simple matter to compute the travel times to the added six source points that bracket the trial hypocenter. The arrival time derivatives with respect to the hypocentral co-ordinate ( $x, y$ and $z$ ) change are then computed as the arrival time difference between the two opposite added sources along each axis, divided by the length between them. The sign and magnitude of these derivatives determine the hypocentral parameter updates.

The arrival time derivative with respect to the origin time change, another element of the Jacobian matrix, is equal to unity. The power of the MSPM is that ray tracing and travel time computations do not have to be recalculated for every iteration. They need only be done once, because the velocity model does not change. With an updated source position in a given cell, one then simply searches the neighboring grid points (for which travel times have been previously computed for each receiver) and interpolates to obtain the theoretical times to both the current trial hypocenter and six additional candidate source positions bracketed the current trial hypocenter, and from these form the updated Jacobian matrix. The procedure is especially useful if many events are to be located for the same velocity model in one simultaneous operation (run of the program) because the receivers are common to all earthquakes being located, and therefore the time calculations from each receiver to all grid points (candidate hypocenter positions) in the velocity model need be done only once. One need only identify the relevant surrounding grid points for each trial hypocenter, extract the relevant times and form the new Jacobian matrix for each iteration. Such an approach is highly computationally efficient and the CPU time is mainly dependent on the inversion process.

A modification of the multiple earthquake location approach just described but with a different physical meaning is to place numerous trial hypocenters for a single earthquake location at a certain spatial sampling across the entire model volume. And then use the local search inversion procedure to find iterative solutions for each trial location simultaneously. The one yielding the overall minimum in the misfit function (or RMS travel time error) would then be chosen as the best (or optimal) solution. In other words, the final solution is obtained by sampling the model space with different length scales to start the inversion process with different initial points 
(trial source parameters), bracketing most local minima and most likely including the global minimum in the RMS error distribution. Note that the global minimum RMS error criterion only makes mathematical sense in the inversion; there is no guarantee that a physical solution will be found. From a practical point of view, it is advisable that the RMS error distribution in the inversion be fully explored to further improve the location results and get an optimal solution.

The multiple events in the MEL method of Bai and Greenhalgh (2006) are simply replaced by multiple initial source candidates in the global-local hybrid solution, with bent ray travel times known from every receiver to every grid node on the model by means of the MSPM ray tracer. The additional candidate source positions can be treated as extra nodes and contribute negligible extra time to the ray tracing. The methodology can be summarized as follows: (1) Use different scales (or lengths or some kinds of à priori information) to sample the solution space (or in the practical sense a small portion of the model space around the station having the minimum arrival time) to obtain a series of initial source parameters to cover any nearby local minima in the model space; (2) Treat these initial source parameters as the multiple source parameters in the MEL procedure, and conduct the matrix inversion as the MEL procedure and stop when the RMS residual of the travel times converges to a pre-determined satisfactory level; (3) Find a best (or optimal) solution with the minimum RMS travel time residual among all the solutions; (4) Observe the RMS error pattern to further improve the location accuracy.

\section{Synthetic examples}

The 3D velocity model as shown in Figure 2, has horizontal dimensions of $40 \mathrm{~km} \times 40 \mathrm{~km}$ and a vertical extent of $14 \mathrm{~km}$. The velocity distribution is smooth and $4.0 \mathrm{~km} / \mathrm{s}$ everywhere along the top surface of the model $(z=0.0 \mathrm{~km})$. At the base of the model $(-14 \mathrm{~km}$ depth $)$ the velocity oscillates between $5.47 \mathrm{~km} / \mathrm{s}$ and $6.03 \mathrm{~km} / \mathrm{s}$, with the maxima and minima $10.0 \mathrm{~km}$ apart. The multiple velocity highs and lows within the horizontal plane either increase or decrease from the center outward (Figure 2a). The velocity in the vertical direction increases linearly with depth (Figure 2b). The variable velocity patterns are the same at the different depths (except for the $z=0 \mathrm{~km}$ plane), but the amplitude of the variations is increasing with depth.
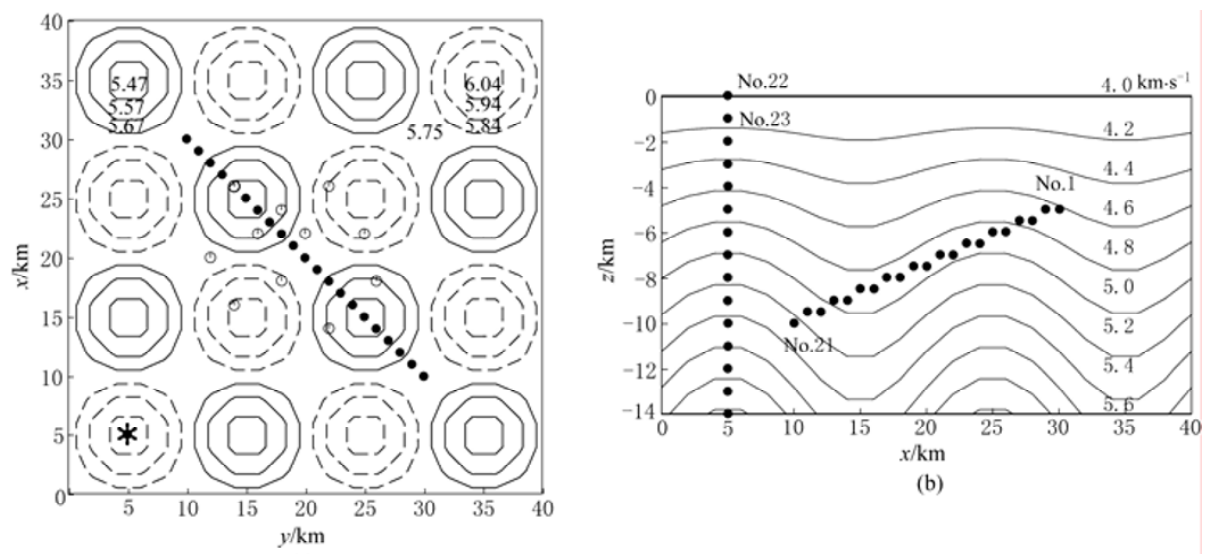

(b)

(a)

\begin{abstract}
Figure 2 Velocity model and source-receiver layout. (a) A plan view of the velocity model in the horizontal plane $z=-14.0 \mathrm{~km}$. Dashed contours denote a velocity high, i.e., a central high and decreasing values outwards; the continuous contours denote a velocity low i.e., a central low and increasing values outwards. Open circles denote surface seismic array projected to the $z=-14.0 \mathrm{~km}$ plane; solid circles denote cross source line projected to the $z=-14.0 \mathrm{~km}$ plane; the black star denotes the location of a vertical source line in horizontal plane; (b) The velocity model in $x$-z cross-section $(y=4.0 \mathrm{~km})$. Solid circles are numbered 'vertical and cross sources' projected to the $x-z$ plane.
\end{abstract}

To simulate 3-D local earthquake location (noise free case), we place 11 receivers on the top central region of the model, and 36 sources within the model. The stations are distributed somewhat irregularly, while the sources are arranged in two different lines. The 'cross line' (consisting of 21 sources, numbered 1 to 21) runs diagonally across the model beneath the recording array (to simulate a dipping fault line) with focal depth 
increasing gradually $(0.5 \mathrm{~km}$ depth interval for every two sources) from the northwest (depth $z=-5 \mathrm{~km}$ ) to the southeast (depth $z=-10 \mathrm{~km}$ ). The 'vertical source line' (compromising 15 sources, numbered 22 to 36 ) is at the position $(x=5.0 \mathrm{~km}, y=5.0 \mathrm{~km})$. It lies entirely outside the seismic station network, with source depth increasing (1.0 km depth interval) from the top surface $(z=0.0 \mathrm{~km})$ to the model base $(z=-14.0 \mathrm{~km})$ (see Figure 2 for details). A random noise time series (absolute maximum value is $10.0 \mathrm{~s}$ ) is added to the calculated theoretical travel times according to the source order. The random noise values constitute the origin times for each source. Such an earthquake source distribution in the complex 3-D model shown would present a real challenge to most hypocenter determination schemes.

\subsection{Accuracy improvement with dense initial source parameters}

In order to test the performance of the hybrid global-local inversion approach (HGLI) to find the optimal solution with location uncertainty, we examine the RMS error distribution, instead of selecting the best solution among the total results after the inversion. Theoretically if the solution space is sufficiently sampled, it is possible to seek a global solution with the HGLI approach. To do this we start with different numbers of initial source locations to conduct the inversion procedure. Here we simply divide the velocity model uniformly into different numbers $(1,8,27,64,125,216$, 343 and 512, note that random selection is also possible) of cubic cells and select the centre of each cubic cell as an initial source location to conduct the inversion (the origin time is set to $0.0 \mathrm{~s}$ in all cases). Figures 3 to 4 show one example, for source No. 28 (located at $x=5.0$ $\mathrm{km}, y=5.0 \mathrm{~km}, z=-6.0 \mathrm{~km}$ and $\left.t_{0}=5.011 \mathrm{~s}\right)$, of the RMS travel time error distribution in two different views $(x-z$ for Figure 3 and $x-y$ for Figure 4) after 10 iterations.

The RMS error exhibits a linear pattern in horizontal (plane) view (Figure 4), but an elliptical pattern in vertical (cross-section) view (Figure 3) with the RMS value reaching a minimum towards the center of the line or the turning point of the ellipse and then increasing in the outward directions. Inspecting Figures 3 and 4, there is a large distance error when only one initial source location (at the centre of the model) is used in the inversion process (see Figures $3 \mathrm{~b}$ and $4 \mathrm{~b}$ ). Clearly the solution depends on the initial source parameters within a fixed velocity field. With a small number of initial source positions applied in the inversion process the solution obtained from the RMS error examination exhibits an improved pattern but still has a moderate solution uncertainty (see Figures $3 \mathrm{c}$ and $4 \mathrm{c}$ ). With an increased number of initial sources, the solution uncertainty is well controlled (see diagrams $\mathrm{d}-\mathrm{i}$ in Figures 3 to 4). For the particular velocity model and station distribution under consideration we find that 27 (or more) initial source positions are sufficient to seek a good optimal solution.

In order to know how to use the RMS error pattern to reduce the solution uncertainty, we give another two examples (here for clarity presentation only 27 initial sources were used). Figure 5 gives the location results for source number 34 located in the vertical source line
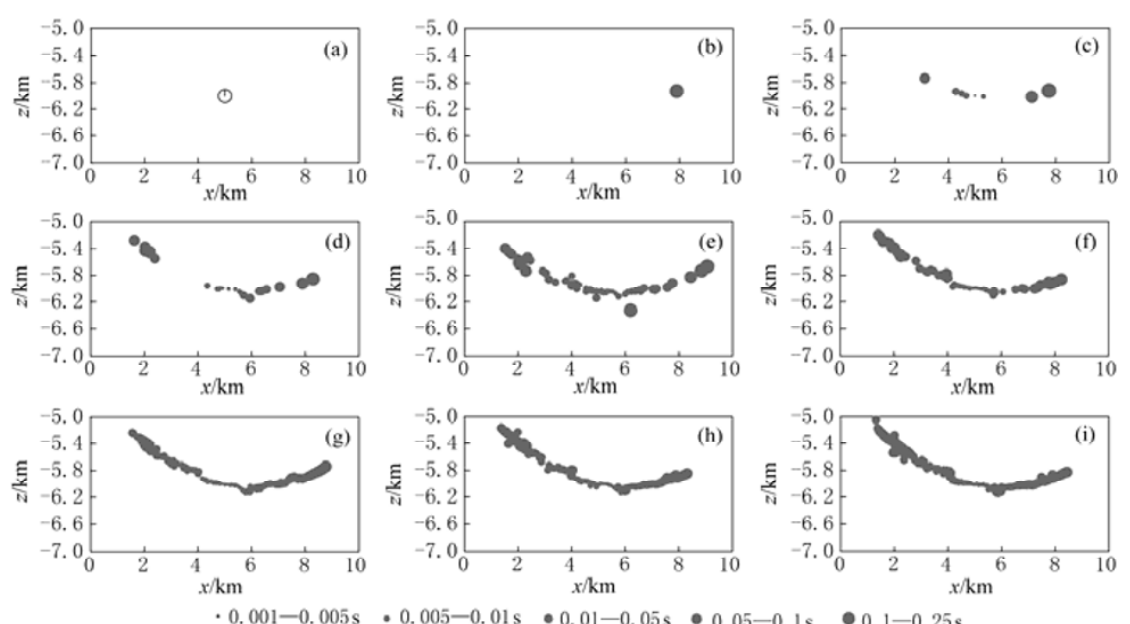

Figure 3 The distributions of RMS travel time error in cross-section view using the HGLI method with an increasing the number of initial sources. In Figures 3 and 4, (a) corresponds to the real source location and (b-i) are the results obtained with $1,8,27,64,125,216,343$ and 512 trial initial sources, respectively. 

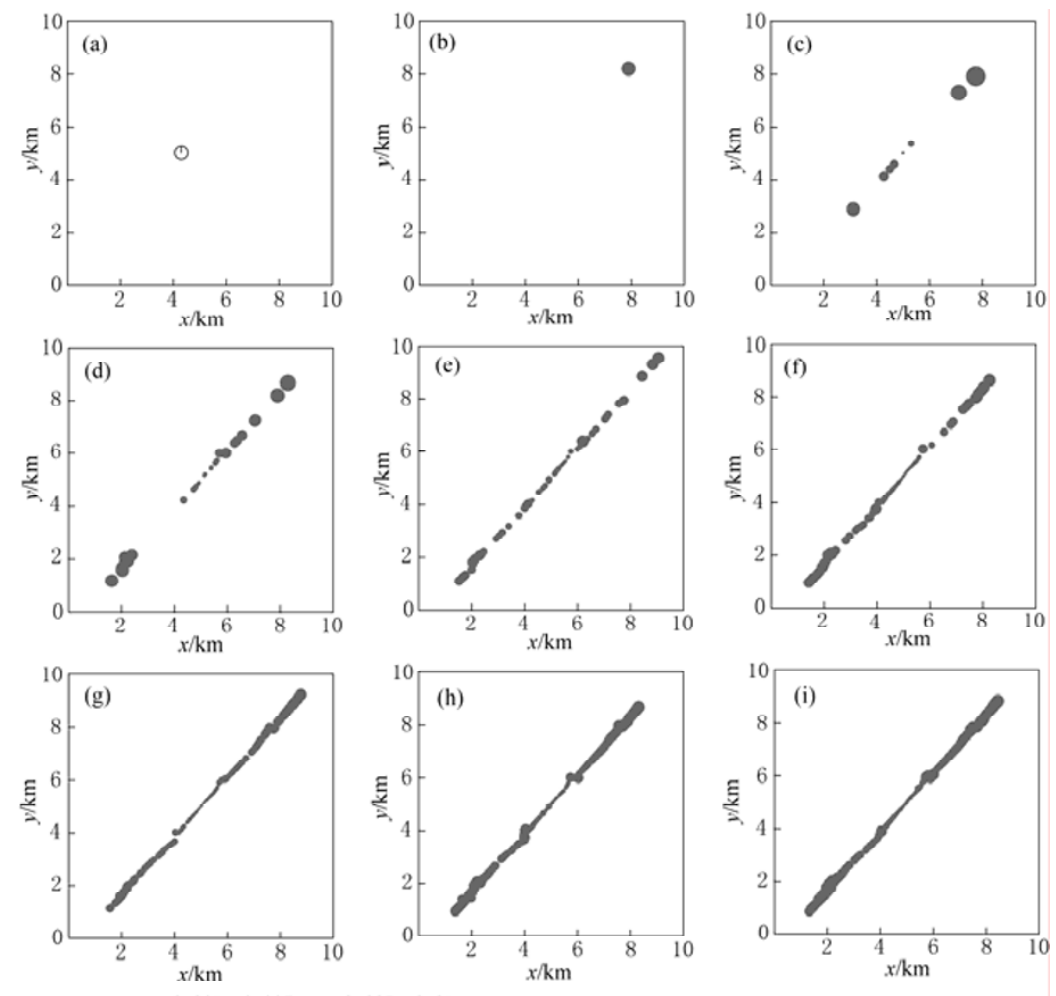

Figure 4 One example of the RMS travel time error distributions in horizontal plan view using the HGLI method with an increasing the number of initial sources.
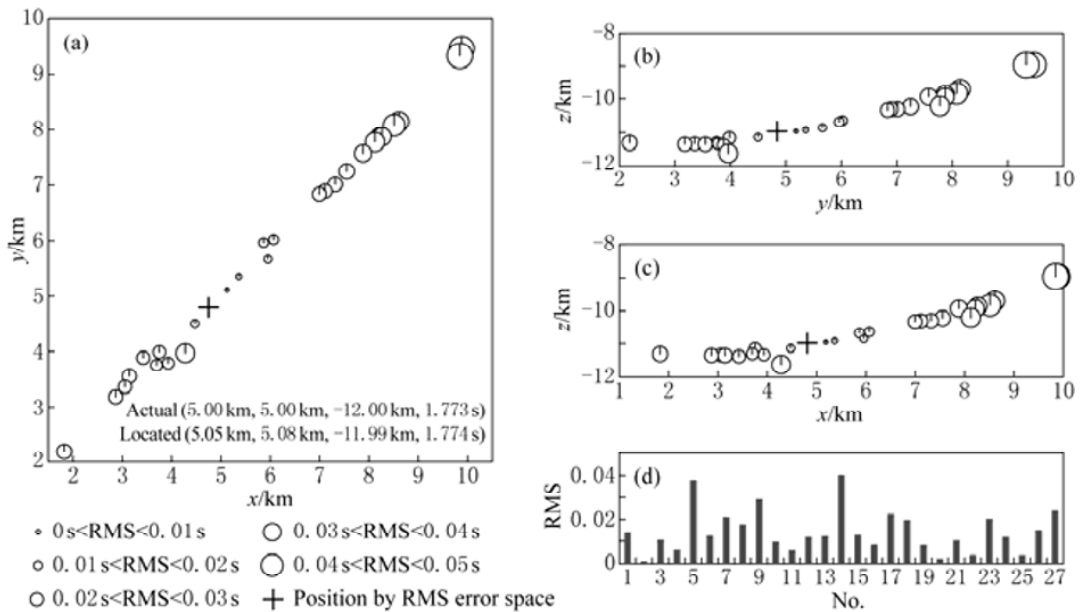

Figure 5 RMS error distributions for the located source (No. 34) with the 27 initial sources in plane and cross-sectional views. (a) shows the results in the $x-y$ plane while (b) and (c) give results in the $y-z$ and $x$-z planes, respectively; (d) gives the RMS error bars in the final solutions using the different initial source locations. The real (true) and located source parameters obtained by the global minimum RMS error criterion are also indicated.

$\left(x=5 \mathrm{~km}, y=5 \mathrm{~km}, z=-12 \mathrm{~km}\right.$ and $\left.t_{0}=1.773 \mathrm{~s}\right)$. The true source position is bracketed by the two opposing lines in plane view (or arcs in cross-section view) with deceasing RMS travel time error toward the actual source position. In such a case the location determined by inspect- ing the RMS error distribution is better than the solution obtained using only the global minimum RMS criterion (see Figures 5a, 5b and 5c).

Figure 6 gives another set of location results for source number 24 located in the vertical source line $(x=5$ 
$\mathrm{km}, y=5 \mathrm{~km}, z=-2 \mathrm{~km}$ and $\left.t_{0}=1.42 \mathrm{~s}\right)$. The global minimum RMS error criterion yields the best location $\left(x=5.05 \mathrm{~km}, y=5.07 \mathrm{~km}, z=-2.04 \mathrm{~km}\right.$ and $\left.t_{0}=1.45 \mathrm{~s}\right)$. Actually, observing the RMS error distribution can improve the solution accuracy when the number of initial sources is not enough to sample the solution space adequately, as discussed above where only 27 initial sources were applied in the inversion process. But by increasing the number of initial sources further the global minimum selection criterion yields an approximately equal solution as RMS error inspection.
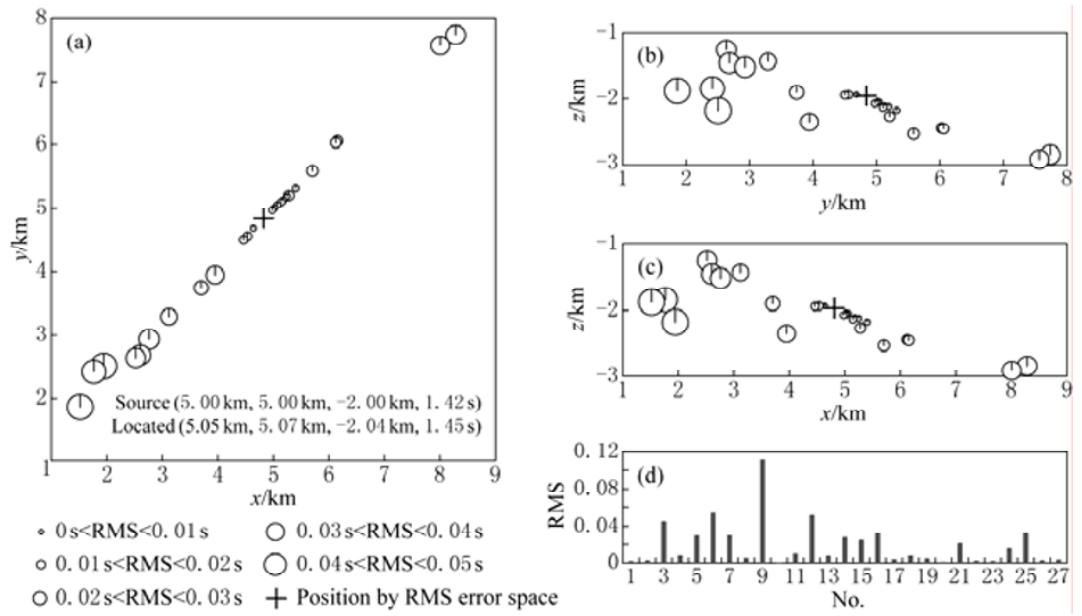

Figure 6 RMS error distributions for the located source (No. 24) in plane and cross-sectional view. See the caption to Figure 5 for an explanation of each diagram.

\subsection{Benchmark tests against the global grid search methods}

It is important to make a comparison between the newly developed approach and the well-known hypocenter determination schemes. Here we choose two of the global optimal solution methods as the reference solutions. One is the direct grid search method (DGS, Nelson and Vidale, 1990); the other is the oct-tree important sampling method (OTIS, Lomax and Curtis, 2001). To minimize CPU time in practical applications, both of the above methods need to calculate the theoretical travel times for the seismic network to every grid point in the velocity model in advance and save them in the form of a numerical travel time table. The principle of the DGS method is first to locate a point with the lowest misfit function (or RMS error) in a coarse grid-model that is thought to contain the earthquake foci, and secondly for each set of travel times from a particular event, the location that produces the smallest travel time residuals (or RMS error) in the refined local grid volume is found.

The OTIS method uses recursive subdivision and sampling of cells in the solution space to generate a cascade of the sampled cells, where the density of sampled cells follows the low RMS travel time error values of the cell center. The procedure is initialized by a global sampling of the full solution space on a coarse regular grid, and the travel time RMS error at the centre of each cell is determined and then the cell with the lowest RMS error is found; secondly the cell with the lowest RMS error is divided into eight child-cells and hence the lowest RMS error among these eight child cells is obtained. The process is repeated until a predetermined numbers of evaluations are completed or other termination criterion is reached.

Figure 7 shows one example for source No. 34 $\left(x=5.0 \mathrm{~km}, y=5.0 \mathrm{~km}, z=-12.0 \mathrm{~km}\right.$ and $\left.t_{0}=0.414 \mathrm{~s}\right)$ of the travel time RMS error distribution in three different views ( $x-y, x-z$ and $y-z)$ using the above DGS method. In the RMS error examination, the solution can be well constrained to find a global solution.

Figure 8 displays one example for source No. 26 $\left(x=5.0 \mathrm{~km}, y=5.0 \mathrm{~km}, z=-4.0 \mathrm{~km}\right.$ and $\left.t_{0}=2.508 \mathrm{~s}\right)$ of the sampling points obtained by the OTIS method. The best solution can also be found by examining the RMS error distribution (Figure 9).

For effective comparison purposes, all three approaches used the same cell length of $1.0 \mathrm{~km}$ in constructing the theoretical traveltimes at all primary nodes in the model, which were then saved in the form of a travel time table. The travel times at other locations inside the cells were computed by tri-linear interpolation, 
linking to the travel time values at the primary nodes. For the DGS method a refined grid spacing of $0.1 \mathrm{~km}$ was used, and for the OTIS method, the termination criterion on the search process was that it be stopped when the length of the child cell reaches $0.1 \mathrm{~km}$. For our new HGLI method, the inversion process is simply terminated after 10 iterations. Table 1 shows the average lo- cation errors for all 36 sources between the three different approaches based on the lowest travel time RMS error. From the table we see that the three approaches perform almost equally well in the sense of location accuracy. For the global search methods (DGS and OTIS) the location accuracy can be further improved if the refined length of local grid is decreased, but CPU time

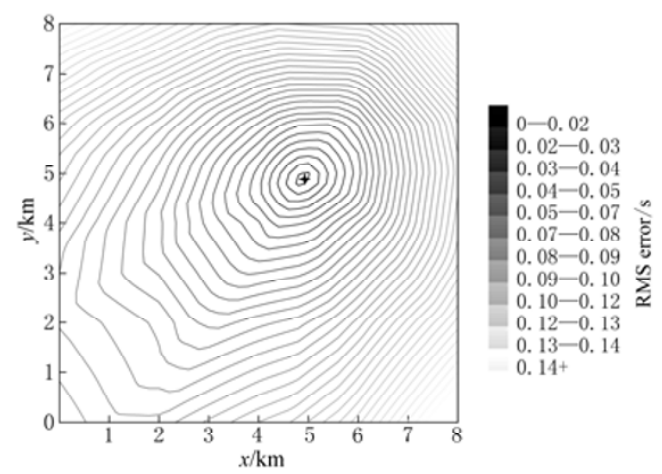

(a)

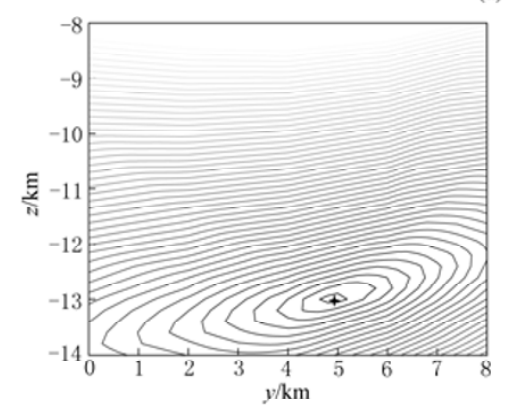

(b)

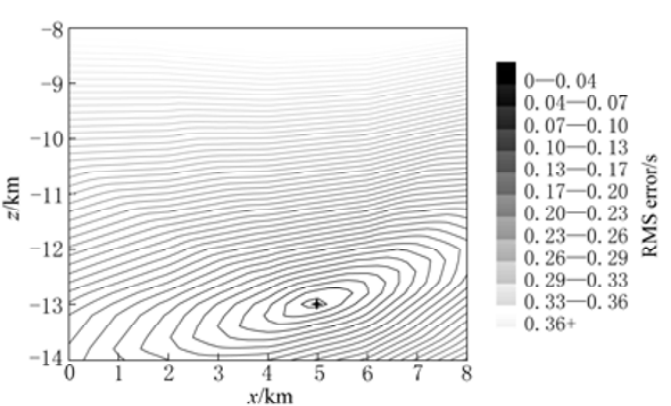

(c)

Figure 7 The RMS travel time error elliptical contours for one example (source No. 34) obtained by the DGS method. Diagram (a) shows results in horizontal (plane) view, while diagrams (b) and (c) in cross-sectional $(x-z, y-z)$ view.

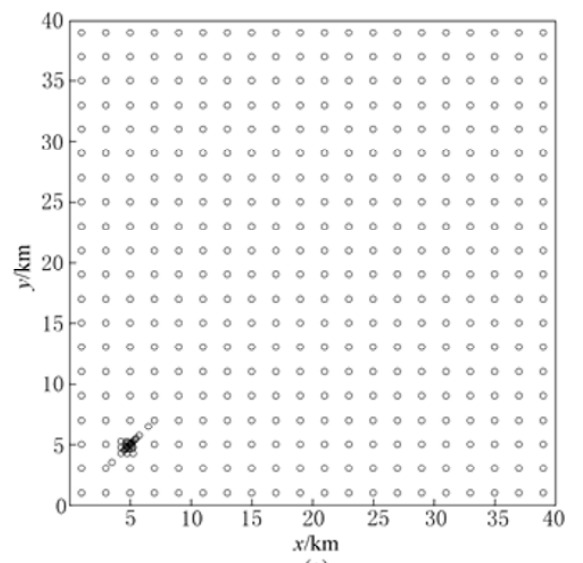

(a)

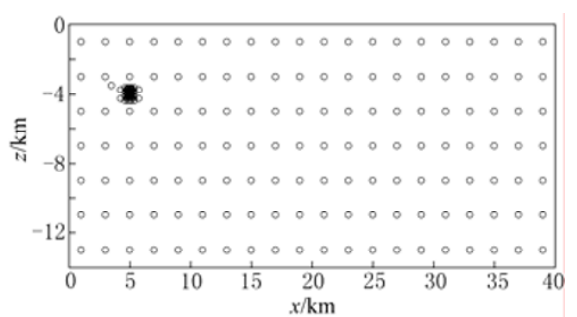

(b)

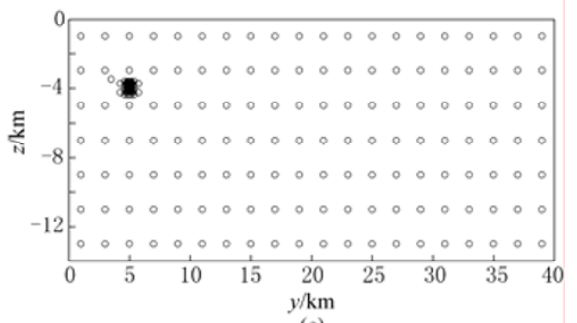

(c)

Figure 8 One example (source No. 26) of the sampling locations used by the OTIS method. Three views are shown in $x-y(\mathrm{a}), x-z$ (b) and $y-z$ planes (c). 


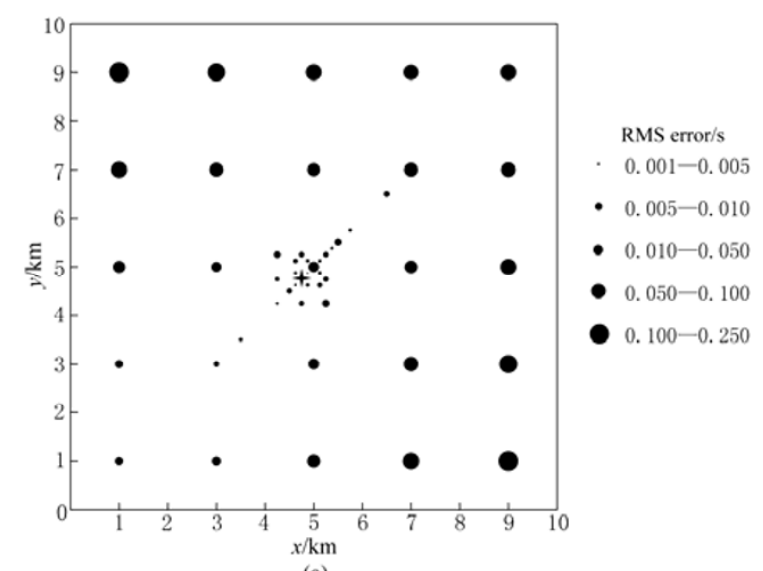

(a)
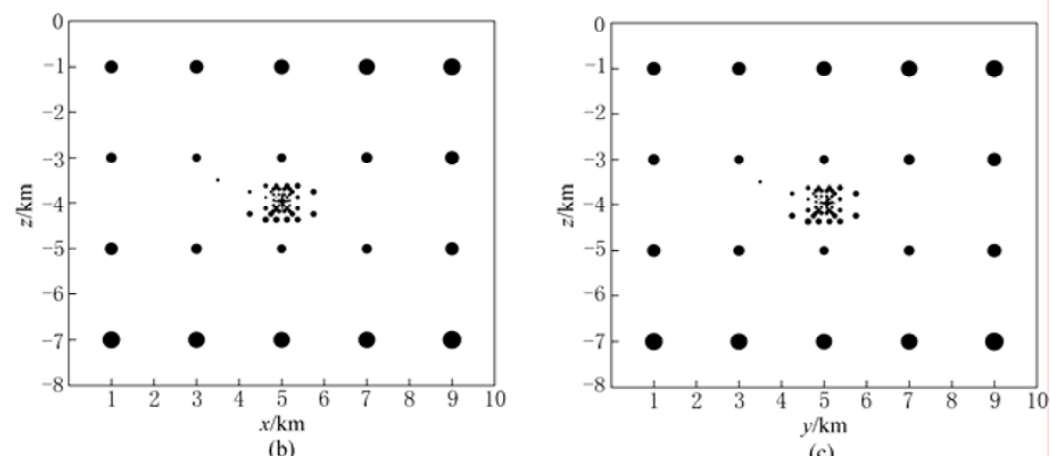

(b)

(c)

Figure 9 One example (source No. 26) of the local RMS travel time error distributions with the OTIS method. Results are given in plan view (a) as well as in cross-sectional views (b, c).

Table 1 Comparison of average location errors for 36 sources with three different approaches

\begin{tabular}{cccccc}
\hline \multirow{2}{*}{ Location methods } & \multicolumn{3}{c}{ Average location errors } \\
\cline { 2 - 5 } & $d_{x} / \mathrm{km}$ & $d_{y} / \mathrm{km}$ & $d_{z} / \mathrm{km}$ & 0.056 & $d_{D} / \mathrm{km}$ \\
DGS & 0.050 & 0.050 & 0.114 & 0.066 & 0.112 \\
OTIS & 0.049 & 0.049 & 0.031 & 0.090 \\
HGLI & 0.049 & 0.056 & 0.0023 & 0.034 \\
\hline
\end{tabular}

Note: The quantities $d_{x}, d_{y}$ and $d_{z}$ are the distance errors in the $x, y$ and $z$ directions, respectively. $d_{D}$ and $d_{t}$ are the hypocentral distance error and travel time error, respectively. The RMS error stands for the root mean square residuals of the travel times (observed minus calculated) at the 11 receivers.

increases accordingly. This aspect will be discussed in the next section.

\subsection{Benchmark tests against the MEL method}

To compare the accuracy between the MEL method and the HGLI method, we present in Table 2 the average location error for all 36 sources using each method. For the MEL procedure two different initial source selection methods (random and intelligent guess) were employed. The first approach involving random selection of one initial source is simply to place it at the geometric center of the model. The second approach involving intelligent guess is based on the relative arrival time patterns. One initial (trial) source is simply placed near the minimum arrival time station. For the HGLI procedure there are also two different approaches. One uses the solution corresponding to the lowest RMS error from the various trial source local inversions. The other is based on examination of the RMS error distribution to find the optimal earthquake location.

Of the four approaches the MEL (random) approach yields the largest errors, but they are still quite reasonable. The situation improves by a factor of 2 to 3 when the MEL (guess) approach is applied. The new HGLI approach results in a further factor of three reductions in location error. With a global selection for the initial source parameters, which cover almost all possible local minima, the solutions are highly accurate. Furthermore with an examination of the RMS error distribution the solution will well be constrained in a global sense (Table 1). 
Table 2 Comparison of average location errors for 36 sources with three different selections of the initial source positions and the RMS error examination

\begin{tabular}{|c|c|c|c|c|c|}
\hline \multirow{2}{*}{ Location methods } & \multicolumn{5}{|c|}{ Average location errors } \\
\hline & $d_{x} / \mathrm{km}$ & $d_{y} / \mathrm{km}$ & $d_{z} / \mathrm{km}$ & $d_{D} / \mathrm{km}$ & $d_{t} / \mathrm{s}$ \\
\hline MEL-random & 0.370 & 0.410 & 0.170 & 0.592 & 0.0128 \\
\hline MEL-guess & 0.119 & 0.165 & 0.114 & 0.264 & 0.0060 \\
\hline GHLI & 0.049 & 0.056 & 0.031 & 0.090 & 0.0023 \\
\hline Error distribution & 0.042 & 0.049 & 0.028 & 0.082 & 0.0020 \\
\hline
\end{tabular}

Note: The symbols are as defined in Table 1. For the methods 'random' stands for the random initial source selection in the MEL procedure, the 'guess' stands for the intelligent selection of the initial source in the MEL procedure, the 'global-local' stands for the global selection of initial source positions as described in this paper, and the 'error distribution' represents the location by examination of the RMS error pattern.

\subsection{Computational efficiency among the three ap- proaches}

As discussed in the introduction, for earthquake early warning, tsunami early warning and rapid hazard assessment and emergency response after strong earthquake occurrence, CPU time is critical factor for selection of the hypocenter determination method. For this reason it is necessary to estimate the computational efficiency among different location approaches so as to meet the minimum time requirement for the above circumstance. For all three methods the forward modeling can be produced to form a theoretical travel time table within a coarse grid velocity model, therefore the CPU time is spent on the global search process or inversion process, including the time taken in the interpolation for travel times on the refined grid. With the three approaches implemented on an IBM Labtop-1.86 GHz, the DGS method took $64.49 \mathrm{~s}$. This was with 64 original cells included in the refined model. The expansion was by two cells outward in all three directions to build up a local refined model. By contrast the OTIS method took $57.85 \mathrm{~s}$ after the child-cell size is reached at $0.1 \mathrm{~km}$. The new HGLI method required only $0.98 \mathrm{~s}$ (10 iterations) to find a good solution. Note that the global search method (DGS and OTIS) will consume even more time when the length of local model is enlarger or the size of the refined grid is further reduced. For example, the DGS method with a local refined model comprising 216 original cells (that is, expanding three cells outward in all three directions) took $243.19 \mathrm{~s}$ to find a global solution. The OTIS method is advantageous over the DGS method because with the latter it is possible to get trapped a local minimum when the refined local model is not large enough or if the first located coarse point is not close to the real source in a complex velocity model. The new HGLI method is the best choice among the three approaches in the sense of computational efficiency and solution accuracy, providing there are suffi- cient initial source locations to sample the complex solution space. Moreover, the RMS error examination is able to find an optimal solution in general.

\subsection{Sensitivity to data noise and location uncer- tainty}

Good earthquake location software should be much more dependent on the accuracy of the velocity model estimation, than on the quality of the arrival time data, on the condition that the noise is not too severe. Or stated another way, it should be sensitive to the model perturbation, but not easily influenced by the noise in the data. To analyze the sensitivity of the location procedure to the data error and location uncertainty, we added random noise to the simulated arrival times. Different levels of random noise were trialed, having maximum and minimum values of $\pm 0.05, \pm 0.1, \pm 0.2$ and $\pm 0.5 \mathrm{~s}$ (that is absolute maximum values of $0.1,0.2,0.4$ and $1.0 \mathrm{~s}$ ). This was to simulate picking errors in the arrival times. All three methods (DGS, OTIS and HGLI) were tested at these four different levels of random noise. The same model, the same hypocenter and the same station layout were used as with the earlier noise-free cases. The located source positions are displayed in three different views $(x-y, x-z$ and $y-z)$ in Figure 10 for the DGS method, in Figure 11 for the OTIS method and in Figure 12 for the HGLI method. Table 3 gives the averaged location errors for all three approaches and for all four levels of noise data. In every case the HGLI method produces the best solution. The OTIS method yields more accurate hypocenter than does the DGS method (see Figures 10-12 and Table 3). If a location error of less than $1.0 \mathrm{~km}$ is tolerable for this sized model, then the global search method (DGS and OTIS) is valid for random noise levels up to $0.2 \mathrm{~s}$, while the new HGLI method performs well even for noise levels as high as $0.4 \mathrm{~s}$. From Table 3 we see that for the global search method (DGS and OTIS) the location errors are doubled when the level of random noise is doubled, but with the 
Table 3 The average location errors using the three different approaches, for different levels of noise data

\begin{tabular}{|c|c|c|c|c|c|c|}
\hline Location methods & Noise level/s & $d_{y} / \mathrm{km}$ & $d_{x} / \mathrm{km}$ & $d_{z} / \mathrm{km}$ & $d_{D} / \mathrm{km}$ & $d_{t} / \mathrm{s}$ \\
\hline DGS & 0.1 & 0.3194 & 0.3417 & 0.0192 & 0.5720 & 0.0084 \\
\hline OTIS & 0.1 & 0.2309 & 0.2274 & 0.0220 & 0.4801 & 0.0209 \\
\hline HGLI & 0.1 & 0.1493 & 0.1549 & 0.0174 & 0.2690 & 0.0027 \\
\hline The best & & HGLI & HGLI & HGLI & HGLI & HGLI \\
\hline DGS & 0.2 & 0.5861 & 0.6139 & 0.0298 & 0.9984 & 0.0139 \\
\hline OTIS & 0.2 & 0.3187 & 0.3369 & 0.0568 & 0.5485 & 0.0247 \\
\hline HGLI & 0.2 & 0.2899 & 0.3108 & 0.0288 & 0.4373 & 0.0061 \\
\hline The best & & HGLI & HGLI & HGLI & HGLI & HGLI \\
\hline DGS & 0.4 & 1.1417 & 1.1861 & 0.0741 & 2.0905 & 0.0367 \\
\hline OTIS & 0.4 & 0.6285 & 0.6215 & 0.0728 & 1.0906 & 0.0467 \\
\hline HGLI & 0.4 & 0.4492 & 0.4709 & 0.0293 & 0.8088 & 0.0195 \\
\hline The best & & HGLI & HGLI & HGLI & HGLI & HGLI \\
\hline DGS & 1.0 & 2.3806 & 2.2583 & 0.1634 & 3.9383 & 0.0781 \\
\hline OTIS & 1.0 & 1.5365 & 1.6406 & 0.1908 & 2.6273 & 0.1126 \\
\hline HGLI & 1.0 & 0.7818 & 0.8166 & 0.0424 & 1.6182 & 0.0353 \\
\hline The best & & HGLI & HGLI & HGLI & HGLI & HGLI \\
\hline
\end{tabular}

Note: The quantities $d_{x}, d_{y}, d_{z}, d_{D}$ and $d_{t}$ are as defined in the caption for Table 1 .
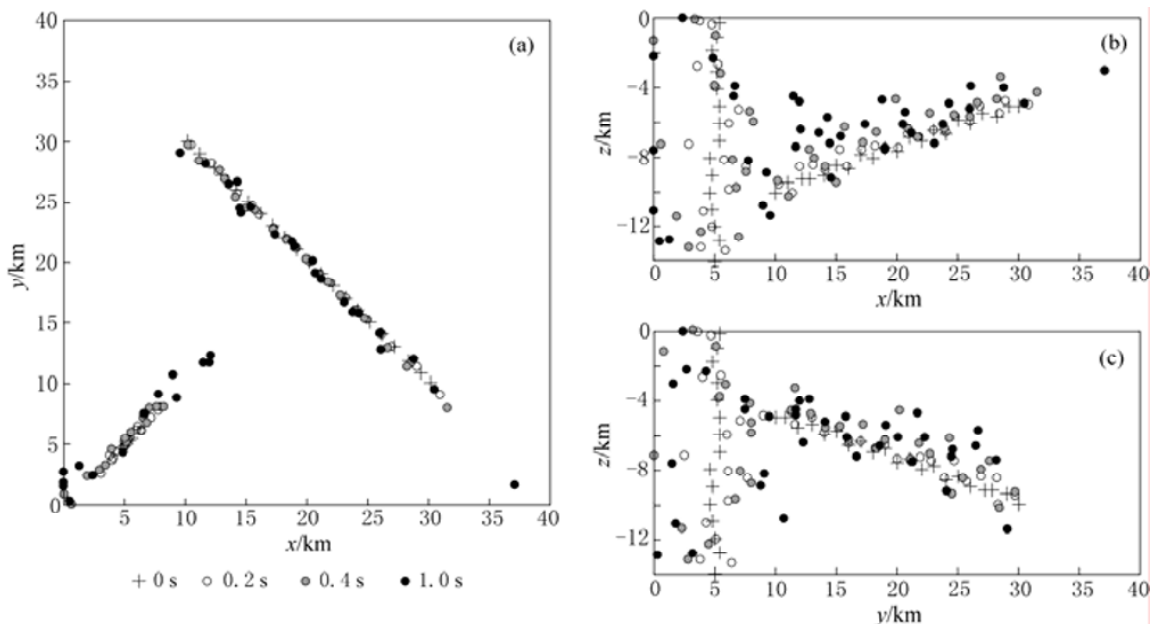

Figure 10 Located source positions in three different views (a: $x-y$; b: $x-z$ and $\mathrm{c}: y-z)$ with the DGS method. In Figures 10-12 the different symbols correspond to the results of different levels of noise data.
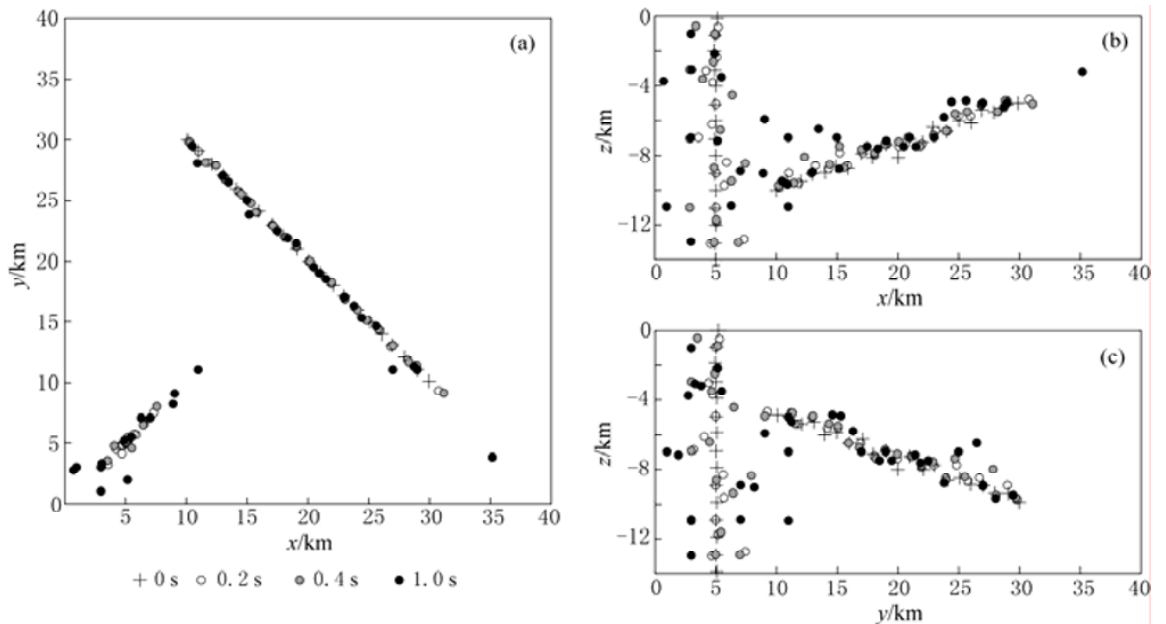

Figure 11 Located source positions in three different views with the OTIS method. 

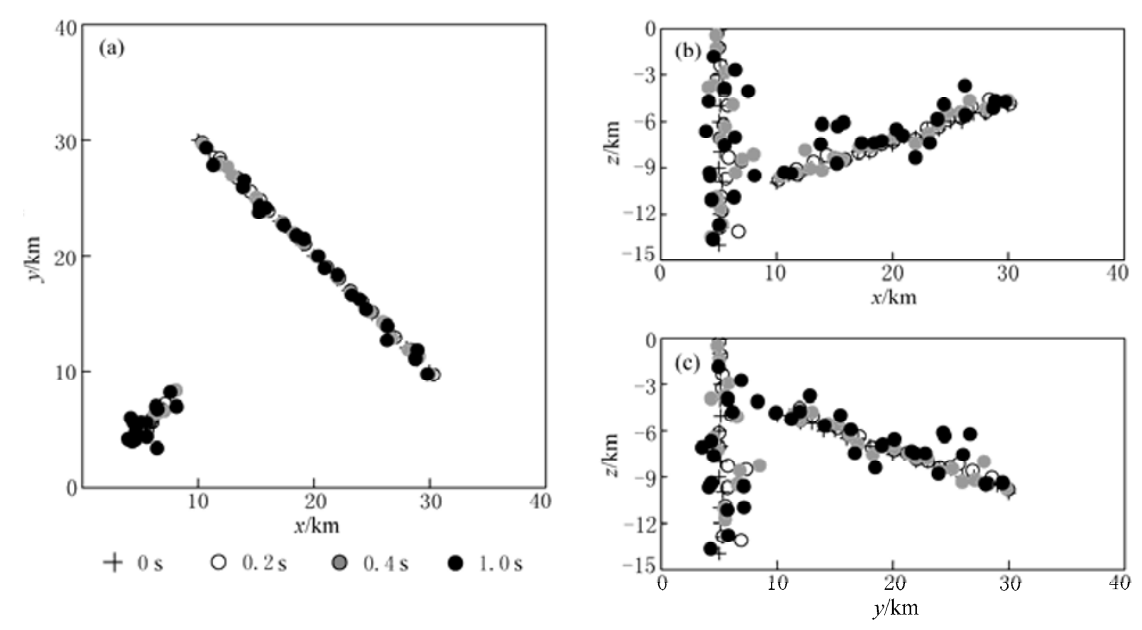

Figure 12 Located source positions in three different views with the new HGLI method.

HGLI approach the location errors increase at a lesser rate. Note that the theoretical travel times for this sized velocity model range from $2.0 \mathrm{~s}$ to $9.0 \mathrm{~s}$, so a $1.0 \mathrm{~s}$ absolute random noise error is rather large (relative errors are over $10.0 \%)$.

From Figures 10-12 we see that all three approaches perform better when the sources beneath the seismic array than when the sources lie far outside the seismic array, as expected. For the global grid search (DGS and OTIS) method a critical step is first to locate the potential node (or cell), which is thought to be near the true source position. Otherwise it will miss the target, regardless of the refined local model. For the OTIS method the same circumstance will happen when the recursive subdivision follows the wrong lowest misfit function in high levels of noise data. Both search processes follow the simple procedure of having an initially coarse global grid (or cell) search, and then the search is refined on a tighter local grid (or cell). To overcome this deficient one should start with a relatively dense global grid (or cell) search and improve the location uncertainty, which in turn overburdens CPU time as the pure Monte Carlo type global search. For the HGLI approach we start with coarse global initial sampling positions and invert each of these initial positions simultaneously with local simplex. In this inversion process we have $N$ different global search paths to cover the whole model so that it is possible to find the optimal solution.

The results show that the HGLI location approach is not very sensitive to modest levels of random noise in the arrival times, but the hypocenter errors or location uncertainty will linearly increase with increasing magnitude of the random noise. Meanwhile, good model estimation is a critical factor in guaranteeing satisfactory performance in earthquake location. In practical application the location uncertainty due to the different levels of noise data can be improved by the weighted matrix inversion; and the location uncertainty due to the rough model estimation can also be reduced by adding a fixed time correction term to a specified station in the inversion.

\section{Conclusions}

The new earthquake location procedure presented here is a HGLI approach involving minimization search, and it seems very promising in practice. The advantages of the method can be summarized as follows.

1) A sufficient number of initial sources which are able to cover almost all local minima (including the global minimum) in the solution space so that it is possible to obtain a global solution and not get trapped in a local minimum.

2) Computational efficiency is improved by several orders of magnitude over the direct global search methods.

3) The location accuracy is comparable to the direct global search method and it can be improved even further with densely sampled initial source positions by examining the RMS error distribution.

4) The HGLI approach is not sensitive to modest levels of data noise and its performance is superior to the two mentioned global search methods in the presence of noise.

The method developed here is very suitable for earthquake early warning, tsunami early warning, rapid hazard assessment and emergency response after strong 
earthquake occurrence, where even seconds of savings in time can be critical to avert or mitigate disasters. The method may also have application to other geophysical inversion problems, such as in seismic tomography, where the selection of a suitable initial (starting) velocity model is also key factor to guaranteeing an accurate and physical solution.

Acknowledgements This research work was jointly funded by the National Natural Science Foundation of China (No. 40774020) and the Key Research Program from Ministry of Education of China (No. 107137).

\section{References}

Bai C Y and Greenhalgh S A (2006). 3-D local earthquake hypocenter determination with an 'irregular' shortest-path method. Bull Seism Soc Amer 96 2 257-2 268.

Bai C Y, Greenhalgh S A and Zhou B (2007). 3-D ray tracing using a modified shortest-path method. Geophysics 72: T27-T36.

Bai C Y, Tang X P and Zhao R (2009). 2D/3D multiply transmitted, converted and reflected arrivals in complex layered media with the modified shortest path method. Geophy J Int 179: 201-214.

Fallat M and Dosso S (1999). Geoacoustic inversion via local, global, and hybrid algorithms. Acoustical Soc Amer 105: 3219-3 230.

Hartzell S and Liu P (1995). Determination of earthquake source parameters using a hybrid global search algorithm. Bull Seism Soc Amer 85: 516-524.

Hartzell S and Liu P (1996). Calculation of earthquake rupture histories using a hybrid global search algorithm: Applied to the 1992 Landers earthquake, California. Phy Earth Planet Inter 95: 79-99.

Kennett B L N (2006). Nonlinear methods for seismic event location in a global context. Phys Earth Planet Inter 158: 46-54.

Kennett B L N and Sambridge M S (1992). Earthquake location-genetic algorithms for teleseisms. Phys Earth Planet Inter 75: 103-110.

Klein F W (1978). Hypocenter location program-HYPOINVERSE: user's guide to version 1, 2, 3 and 4. US Geol Surv Open-File Rept 694: 1-113.

Klein F W (1989). User's guide to HYPOINVERSE: A program for VAX computers to solve for earthquake location. US Geol Surv Open-File Rept 314: 44.

Lee W H K and Lahr J C (1975). HYPO71 (revised): A computer program for determining hypocenter, magnitude, and first-motion pattern of local earthquakes. US Geol Surv Open-File Rept 311: 1-116.
Lepage G P (1978). A new algorithm for adaptive multi-dimensional integration. J Comput Phys 27: 192-203.

Lienert B R, Berg E and Frazer L N (1986). Hypocenter: An earthquake location method using centered, scaled, and adaptively damped least squares. Bull Seism Soc Amer 76: 771-783.

Lomax A and Curtis A (2001). Fast, probabilistic earthquake location in 3D models using oct-tree importance sampling. Geophys Res Abstr 3: 955, www.alomax.net/nlloc/octtree.

Mosegaard K and Tarantola A (1995). Monte Carlo sampling of solutions to inverse problems. J Geophys Res 100: 12 431-12 447.

Moser T J (1991). Shortest path calculation of seismic rays. Geophysics 56: 59-67.

Nelson G D and Vidale J E (1990). Earthquake location by 3-D finite difference travel times. Bull Seism Soc Amer 80: 395-410.

Pavlis G and Booker J R (1980). The mixed discrete-continuous inverse problem: Application of the simultaneous determination of earthquake hypocenters and velocity structure. J Geophys Res 85(B9): 4 801-4 810.

Rawlinson N and Sambridge M (2004). Multiple reflection and transition phases in complex layered media using the multistage fast marching method. Geophysics 69: 1338-1 350.

Sambridge M (1999a). Geophysical inversion with a neighbourhood algorithm. I. Searching a parameter space. Geophys J Int 138: 479-494.

Sambridge M (1999b). Geophysical inversion with a neighbourhood algorithm. II. Appraising the ensemble. Geophys J Int 138: 727-746.

Sambridge M and Kennett B L N (2001). Seismic event location: Nonlinear inversion using a neighbourhood algorithm. Pure Appl Geophys 158: 241-257.

Sambridge M and Mosegaard K (2002). Monte Carlo methods in geophysical inverse problems. Rev Geophys 40: doi:10.1029/2000RG000089.

Tarantola A (2005). Inverse Problem Theory and Methods for Model Parameter Estimation. SIAM, Philadelphia, 54-57.

Thurber C H (1985). Non-linear earthquake location: Theory and examples. Bull Seism Soc Amer 78: 2 062-2 076.

Thurber C H and Rabinowitz N (2000). Advances in Seismic Event Location. Kluwer, Amsterdam, 1-276.

Vidale J E (1988). Finite-difference calculation of travel times. Bull Seism Soc Amer 78: 2062-2 076.

Vidale J E (1990). Finite-difference calculation of travel time in three dimensions. Geophysics 55: 521-526.

Waldhauser F and Ellsworth W L (2000). Double-difference earthquake location algorithm: Method and application to the northern Hayward fault, California. Bull Seism Soc Amer 90: 1 353-1 368.

Zhou B, Sinadinovski C and Greenhalgh S A (1992). Non-linear inversion travel time tomography: Imaging high contrast inhomogeneities. Explor Geophys 23: 459-469 (in Chinese with English abstract). 